\title{
Epithelial mesenchymal transition induced by the CXCL9/CXCR3 axis through AKT activation promotes invasion and metastasis in tongue squamous cell carcinoma
}

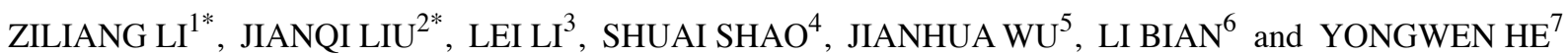 \\ ${ }^{1}$ Department of Oral Implantology, The Affiliated Stomatological Hospital of Kunming Medical University, Kunming, \\ Yunnan 650101; ${ }^{2}$ Department of Stomatology, The Affiliated Yan'an Hospital of Kunming Medical University, \\ Kunming, Yunnan 650051; ${ }^{3}$ Head and Neck Tumor Research Center, The 3rd Affiliated Hospital of \\ Kunming Medical University, Kunming, Yunnan 650118; ${ }^{4}$ Department of Oral and Maxillofacial Surgery, \\ The Second People's Hospital of Yunnan Province, Kunming, Yunnan 650021; ${ }^{5}$ Department of Periodontology, \\ The Affiliated Stomatological Hospital of Kunming Medical University, Kunming, Yunnan 650101; \\ ${ }^{6}$ Department of Pathology, The First Affiliated Hospital of Kunming Medical University, Kunming, \\ Yunnan 650032; ${ }^{7}$ Department of Dental Research, The Affiliated Stomatological Hospital \\ of Kunming Medical University, Kunming, Yunnan 650101, P.R. China
}

Received August 7, 2017; Accepted December 13, 2017

DOI: 10.3892/or.2017.6169

\begin{abstract}
The present study aimed to assess the induction of epithelial-mesenchymal transition (EMT), invasion, and metastasis by the chemokine CXCL9/receptor CXCR3 axis in tongue squamous cell carcinoma (TSCC), unveiling the underlying mechanisms and providing new insights into the prevention and treatment of oral cancer metastasis. The expression levels of CXCL9 and CXCR3 in TSCC tissue specimens were determined by immunohistochemistry, assessing differences between samples with cervical lymph node metastasis and those without. Moreover, protein expression or activity in the TSCC Cal-27 cell line was controlled by
\end{abstract}

Correspondence to: Dr Yongwen He, Department of Dental Research, The Affiliated Stomatological Hospital of Kunming Medical University, Block C 1088 Hai Yuan Road, High and New Techology Zone, Kunming, Yunnan 650101, P.R. China

E-mail: heyongwen2@sina.com

Dr Li Bian, Department of Pathology, The First Affiliated Hospital of Kunming Medical University, 295 Xichang Road, Kunming, Yunnan 650032, P.R. China

E-mail: bianli1976@sina.com

${ }^{*}$ Contributed equally

Abbreviations: AA, amino acids; CAFs, carcinoma-associated fibroblasts; EMT, epithelial-mesenchymal transition; HNSCC, head and neck squamous cell carcinoma; HRP, horseradish peroxidase; LN, lymph node; PI3K, phosphatidylinositol 3 kinase; TSCC, tongue squamous cell carcinoma

Key words: tongue squamous cell carcinoma, CXCL9/CXCR3 axis, EMT, invasion, Akt neutralizing antibodies, gene transfection, or knock-out. Then, alterations of cell proliferation, migration, invasion, and the cytoskeleton were analyzed by CCK-8, cell scratch, Transwell, and cytoskeleton staining assays, respectively. Alterations of EMT markers (E-cadherin and vimentin) in Cal-27 cells were detected by immunofluorescence and western blotting. In addition, western blotting was utilized to detect protein expression levels of Akt2, p-Akt2, eIF4E and p-eIF4E, and to explore the regulatory roles and mechanisms of the CXCL9/CXCR3 axis in invasion and metastasis. Significantly increased expression levels of CXCL9 and CXCR3 were detected in tissue specimens with lymph node metastasis compared with those without $(\mathrm{P}<0.01)$. Overexpression of CXCL9/CXCR3 in Cal-27 cells resulted in cytoskeleton alterations, decreased E-cadherin expression, increased vimentin levels, enhanced migration and invasion $(\mathrm{P}<0.05)$, and increased phosphorylated Akt2 and eIF4E levels $(\mathrm{P}<0.05)$. These results revealed that in TSCC, the CXCL9/CXCR3 axis could activate the Akt signaling pathway, with EMT and cytoskeleton rearrangement, promoting invasion and metastasis.

\section{Introduction}

Oral cancer incidence varies from 1.06 to $1.09 / 100,000$ cases, and is steadily rising worldwide, with the age at onset increasingly younger (1). Tongue cancer is the most common oral malignancy (2), and more than $98 \%$ of cases are found at the anterior lingual $2 / 3$, mostly at the tongue base. In addition, most cases diagnosed are squamous cell carcinoma and seldom adenocarcinoma. Tongue cancer is usually accompanied with early cervical lymph node metastasis, with a high incidence of $40-80 \%$ (3). A 5 -year survival rate of $80 \%$ was documented for patients with no cervical lymph node metastasis; while $30 \%$ was found in those with metastasis (4). 
Invasion and metastasis of tongue squamous cell carcinoma (TSCC) is a comprehensive, multistep and sequential process, which encompasses inducible lymphogenesis and/or angiogenesis, dissemination of tumor cells and their intravasation into lymph or blood vessels, and random or specific residing at the target microvessels. This is followed by extravasation out of vessels, proliferation and invasion into distal tissues and organs, and finally formation of metastatic foci (5). Chemokines are closely related to tumor invasion and metastasis. They bind to specific tumor cell surface receptors and induce cytoskeleton rearrangement, promoting tight attachment of tumor cells to lymphatic endothelial cells and their directional migration (6).

Chemokines and their receptors extensively exist in a wide range of cells, playing essential roles in embryogenesis, hematopoiesis, HIV infection, tumor invasion, and metastasis (7). Chemokines are secretory small molecule proteins of the cytokine superfamily, with chemotactic capabilities $(8,9)$. Chemokine receptors belong to the superfamily of $\mathrm{G}$ protein coupled seven transmembrane receptors (10), whose $\mathrm{N}$-terminal domains bind to specific ligands, with cytosolic domains conjugating with $\mathrm{G}$ protein; phosphorylation of their C-terminal serine/threonine residues contributes to signal transduction. The chemokine receptor CXCR3, including three spliced variants, termed CXCR3-A and CXCR3-B and CXCR3-alt, which belongs to the CXC subfamily of chemokine receptors, is highly expressed in colon cancer, melanoma, B lymphoma and breast cancer cells. Its specific binding to its ligand chemokine CXCL9 mediates the directional migration of cancer cells, regulating cancer invasion and metastasis (11).

The chemokine CXCL9, also termed MIG, is a member of the chemokine $\gamma$-subfamily (CXC family) of proteins. The human CXCL9 gene is located on chromosome 4q21, next to the gene encoding CXCL10 (IFN2 $-\gamma$ inducible $10 \mathrm{kDa}$ protein, IP210). CXCL9 is primarily induced by IFN2- $\gamma$, and produced in lymphocytes, monocytes/macrophages, and fibroblasts. CXCR3 belongs to the CXC chemokine subfamily (12). The human CXCR3 protein has 368 amino acids (AA) and a molecular weight of $41 \mathrm{kDa}(13)$. Its extracellular N-terminal structural domain composed of AA 1-57 contributes to ligand recognition and binding. Chemotaxis by CXCL9 is mainly mediated by CXCR3, which, upon binding to CXCL9, initiates Src phosphorylation and activates Src kinase, concomitantly enhancing the activities of phosphatidylinositol 3 kinase (PI3K) and the downstream Akt (14).

In the present study, high expression levels of the chemokine CXCL9 and its receptor CXCR3 were detected in TSCC tissue samples from patients with lymph node metastasis, indicating the possible involvement of the CXCL9/CXCR3 axis in TSCC invasion and metastasis. Furthermore, CXCL9/CXCR3 was demonstrated to activate the Akt signaling pathway, which possibly mediates epithelial-mesenchymal transition (EMT), thus promoting TSCC invasion and metastasis.

\section{Materials and methods}

Reagents. The EnVision + HPR/DAB kit was purchased from Shanghai Gene Technology Co., Ltd. (Shanghai, China), and the cytokine CXCL9 from PeproTech Inc. (Rocky Hill, NJ, USA). CXCR3 neutralizing antibodies $(2.0 \mu \mathrm{g} / \mathrm{ml}$; mouse, monoclonal; cat. no. 49801) and $\operatorname{IgG}(2.0 \mu \mathrm{g} / \mathrm{ml}$; mouse; cat. no. A7028) were purchased from R\&D Systems (Minneapolis, MN, USA) and Beyotime Biotechnology Co., Ltd. (Shanghai, China), respectively. Anti-E-cadherin (1:200; rabbit, polyclonal; cat. no. ab53226) and anti-vimentin (1:100; rabbit, monoclonal; cat. no. ab16700) were both purchased from Abcam (Cambridge, UK). The phalloidin-labeled cytoskeleton staining kit was purchased from Sigma-Aldrich (St. Louis, MO, USA), the CCK-8 kit from Dojindo Molecular Technologies, Inc. (Kumamoto, Japan), the plasmid extraction kit from Qiagen GmbH (Hilden, Germany), the high efficiency transfection (HET) kit from Shenzhen Biowit Technologies Co., Ltd. (Shenzhen, China), and the RNA reverse transcription kit from Thermo Fisher Scientific (Waltham, MA, USA).

EnVision immunohistochemistry. Ten normal tongue mucosa specimens were used as negative controls. TSCC specimens (provided by Professor Tiejun Li from Peking University Stomatological Hospital) with pathologically definitive diagnosis were collected from 51 cases without preoperative treatment, averaging 22 years old, and including 30 males and 21 females. Among them, 28 cases had lymph node (LN) metastasis while the remaining 23 had no LN metastasis. CXCL9 and CXCR3 expression levels were detected by Envision immunohistochemistry and intracellular brown particles reflected positive signals. The semi-quantitative additive score method was used to quantify positive staining by counting cells in 10 high power fields randomly in every slide. Specific criteria were: i) score based on the percentage of positive cells $(0, \leq 5 \% ; 1,6-25 \% ; 2,26-50 \% ; 3,51-75 \% ; 4,>75 \%)$; ii) score based on signal intensity in most cells (1, slight yellow; 2 , yellow; 3 , brown). The product of the score based on the percentage of positive cells and the signal intensity score were used as the final score: negative, score of $0(-)$; slightly positive, score of 1-4 (+); positive, score of 5-8 (++); strongly positive, score of 9-12 (+++). Double blinded quantification of the slides by 2 people was applied and a difference of 3 points between values required re-examination.

Cell culture. The human TSCC cell line Cal-27 (ATCC; Manassas, VA, USA) was cultured in high-glucose DMEM with $10 \% \mathrm{FBS}$, at $37^{\circ} \mathrm{C}$ and $5 \% \mathrm{CO}_{2}$. Cells at the logarithmic growth phase were used for experiments.

Establishment of an hCXCR3 stable cell line. The hCXCR3 expression vector pLVX-hCXCR3-mCMV-ZsGreen-PGKPuro was constructed by DNA synthesis (1107 bp) with the following primers pLVX-hCXCR3-EcoRI-F, 5'-CCGGA ATTCGCCACCATGGTCCTTGAGGTGAGTGACCACC AAG-3'; pLVX-hCXCR3-BamHI-R, 5'-CGCGGATCCTCA CAAGCCCGAGTAGGAGGCCTCTGAG-3'. Prior to large volume plasmid extraction, the vector was characterized by enzymatic digestion and sequencing. The lentivirus was packaged by infecting $293 \mathrm{~T}$ cells and concentrated. Following purification, Cal-27 cells were infected with the lentivirus under selection by puromycin.

Establishment of an hCXCR3 shRNA stable cell line in the Cal-27 background. The shCXCR3 (pLVX-ShRNA-purohCXCR3) expression vector was constructed by DNA synthesis with the following primers: hCXCR3-F, 5'-GATCCGCCTACT 
GCTATGCCCACATCTTCAAGAGAGATGTGGGCATAG CAGTAGGCTTTTTTCTCGAGG-3'; hCXCR3-R, 5'-AATT CCTCGAGAAAAAAGCCTACTGCTATGCCCACATCTC TCTTGAAGATGTGGGCATAGCAGTAGGCG-3'.

Cell proliferation assay. Cells at the log-phase were trypsinized, seeded in 96-well plates at a density of $1.2 \times 10^{4}$ cells $/ \mathrm{ml}$ in $100 \mu \mathrm{l}$, and cultured overnight at $37^{\circ} \mathrm{C}$. Then, the culture medium was aspirated, and supplemented with $100 \mu \mathrm{l}$ of CXCL9 protein, CXCR3 neutralizing antibodies $(2.0 \mu \mathrm{g} / \mathrm{ml})$, and $\operatorname{IgG}(2.0 \mu \mathrm{g} / \mathrm{ml})$ for $24 \mathrm{~h}$, respectively, followed by the addition of $10 \mu \mathrm{l}$ of CCK- 8 reagent for $3 \mathrm{~h}$. The absorbance at $450 \mathrm{~nm}$ was assessed on a microplate reader. Each experiment was repeated 3 times.

Scratch assay. Cells at the log-phase were seeded in 6-well plates at a density of $5 \times 10^{5}$ cells/well. At a confluency of $95 \%$, $0.5 \mathrm{ml}$ of CXCL9, CXCR3 neutralizing antibodies $(2.0 \mu \mathrm{g} / \mathrm{ml})$ and $\mathrm{IgG}(2.0 \mu \mathrm{g} / \mathrm{ml})$ were added for $3 \mathrm{~h}$. Then, the cell monolayer was scratched with a $200-\mu 1$ pipette tip. Detached or dead cells were washed out with PBS, and serum-free culture media were added. A total of 3 fields/group were photographed under a fluorescence microscope at 12,24 , and $48 \mathrm{~h}$, respectively. Cell migration rates were calculated with ImageJ software 6.0 and each experiment was repeated three times.

Cell invasion assay. Transwell chambers were used for cell invasion assays. The upper chambers were seeded with $0.5 \mathrm{ml}$ of Cal-27 cells in high-glucose complete medium, with the lower chambers filled with $0.7 \mathrm{ml}$ of high-glucose complete culture medium. For each group, three different interventions were applied: i) the lower chamber was supplemented with $100 \mathrm{nM}$ CXCL9; ii) the upper chamber was supplemented with $2 \mu \mathrm{g} / \mathrm{ml}$ CXCR3 neutralizing antibodies in combination with $100 \mathrm{nM}$ CXCL9 protein in the lower chamber; and iii) the upper chamber was supplemented with $2 \mu \mathrm{g} / \mathrm{ml} \mathrm{IgG}$ combined with $100 \mathrm{nM}$ CXCL9 in the lower chamber. After $24 \mathrm{~h}$, the membranes were separated, stained, sealed, and assessed for invasive cells under a microscope. Every experiment was repeated 3 times.

Cytoskeleton staining. Cells under routine culture were supplemented with $1 \mathrm{ml}$ of the CXCL9 protein, CXCR3 neutralizing antibodies $(2.0 \mu \mathrm{g} / \mathrm{ml})$, and $\operatorname{IgG}(2.0 \mu \mathrm{g} / \mathrm{ml})$, followed by $4 \%$ paraformaldehyde fixation for $48 \mathrm{~h}$. Then, $0.5 \mathrm{ml}$ of $40 \mu \mathrm{g} / \mathrm{ml}$ phalloidin-rhodamine solution was added for $60 \mathrm{~min}$ in the dark in a humid box at room temperature, and sealed before analysis by fluorescence microscopy. Edge aggregation of F-actin was assessed in Cal-27 cells.

Cell immunofluorescence staining. Cultured cells were fixed in paraformaldehyde at $4^{\circ} \mathrm{C}$, permeabilized with $0.1 \%$ Triton $\mathrm{X}-100$, blocked with $10 \%$ goat serum, and incubated with anti-E-cadherin (1:200; rabbit, polyclonal; cat. no. ab53226) or anti-vimentin (1:100; rabbit, monoclonal; cat. no. ab16700, both from Abcam) primary antibodies overnight at $4^{\circ} \mathrm{C}$ in the dark, in a humid box. This was followed by incubation with fluorescence-labeled secondary antibody $(2 \mu \mathrm{g} / \mathrm{ml}$, goat anti-rabbit $\operatorname{IgG}(\mathrm{H}+\mathrm{L})$; antibody, cat. no. A11012; Thermo Fisher Scientific) in a shaker incubator. Counterstaining was performed with $100 \mu \mathrm{g} / \mathrm{ml}$ DAPI before analysis by fluorescence microscopy.

Western blotting. Cal-27 cells were lysed on ice with lysis buffer. Proteins in whole cell lysates were separated by SDS-PAGE, electrically transferred onto Immobilon-P membranes, incubated with primary antibodies anti-Akt2 (1:500; rabbit, polyclonal; cat. no. ab8805), anti-p-Akt (1:500; rabbit, polyclonal; cat. no. ab38513), anti-eIF4E (1:500; rabbit, monoclonal; cat. no. ab33766), anti-p-eIF4E (1:1,000; rabbit, monoclonal; cat. no. ab76256; all from Abcam) overnight at $4^{\circ} \mathrm{C}$, followed by $1 \mathrm{~h}$ of incubation with a horseradish peroxidase (HRP)-labeled secondary antibody (1:3,000; goat anti-rabbit IgG-HRP; cat. no. sc2004; Santa Cruz Biotechnology, Inc., Santa Cruz, CA, USA) at room temperature before chemiluminescence detection (Western Chemiluminescent HRP substrate; Millipore, Billerica, MA, USA).

Statistical analysis. The Wilcoxon non-parametric test was applied to assess intergroup differences for enumeration data. One-way ANOVA was used for measurement data; intergroup differences were analyzed using the LSD-t method. $\mathrm{P}<0.05$ indicated a statistically significant difference.

\section{Results}

CXCL9 and CXCR3 levels are higher in TSCC with LN metastasis than in normal tissues or TSCC without LN metastasis. In tongue mucosa epithelial tissue specimens from the 10 normal cases, no CXCL9-positive cells were detected, i.e., CXCL9 (-). In addition, CXCR3-positive cells were observed in individual specimens, and positive cells were limited and primarily located in the basal layer, i.e., CXCR3 (-) (Fig. 1A and B). Of the 51 TSCC specimens, 24 (47.06\%) were CXCL9 (-), $10(19.60 \%)$ CXCL9 (+), $10(19.60 \%)$ CXCL9 (++), and $7(13.73 \%)$ CXCL9 (+++). The 23 TSCC specimens without cervical LN metastasis were mostly CXCL9 $(-) /(+)$, while the 20 out of 28 with cervical LN metastasis were CXCL9 $(+)$ to $(+++)$. Statistical analysis revealed significantly higher CXCL9 expression in TSCC with cervical LN metastasis compared with those without metastasis $(\mathrm{P}<0.01)$ as assessed by the Wilcoxon non-parametric test $(\mathrm{Z}=-2.68, \mathrm{p}=0.0074)$ (Fig. 1C).

Of the 51 TSCC specimens, $10(19.61 \%)$ were CXCR3 (-), 8 (15.69\%) CXCR3 (+), 15 (29.41\%) CXCR3 (++), and 18 (35.29\%) CXCR3 (+++). The 23 TSCC without cervical LN metastasis were mostly CXCR3 (-) to (+), while the 28 with cervical LN metastasis were mostly CXCR3 (++) to (+++); a statistically significant difference $(\mathrm{P}<0.01)$ in $\mathrm{CXCR} 3$ expression in the LN metastasis group was reflected in the Wilcoxon nonparametric test $(\mathrm{Z}=-2.66, \mathrm{P}=0.0079)$ (Fig. 1C).

The CXCL9/CXCR3 axis does not promote proliferation in Cal-27 cells. The hCXCR3 and shCXCR3 expression vectors were constructed (Fig. 2A and B). Cal-27 cells were seeded in 96-well plates and incubated with CXCL9 at 0,50, 100, and $150 \mathrm{nM}$, respectively, for $24 \mathrm{~h}$, followed by $3 \mathrm{~h}$ of incubation with CCK-8 reagent at room temperature before absorbance assessment at $450 \mathrm{~nm}$. Compared with the control group, no statistical difference $(\mathrm{P}>0.05)$ was detected in terms of cell proliferation (Fig. 2C). 

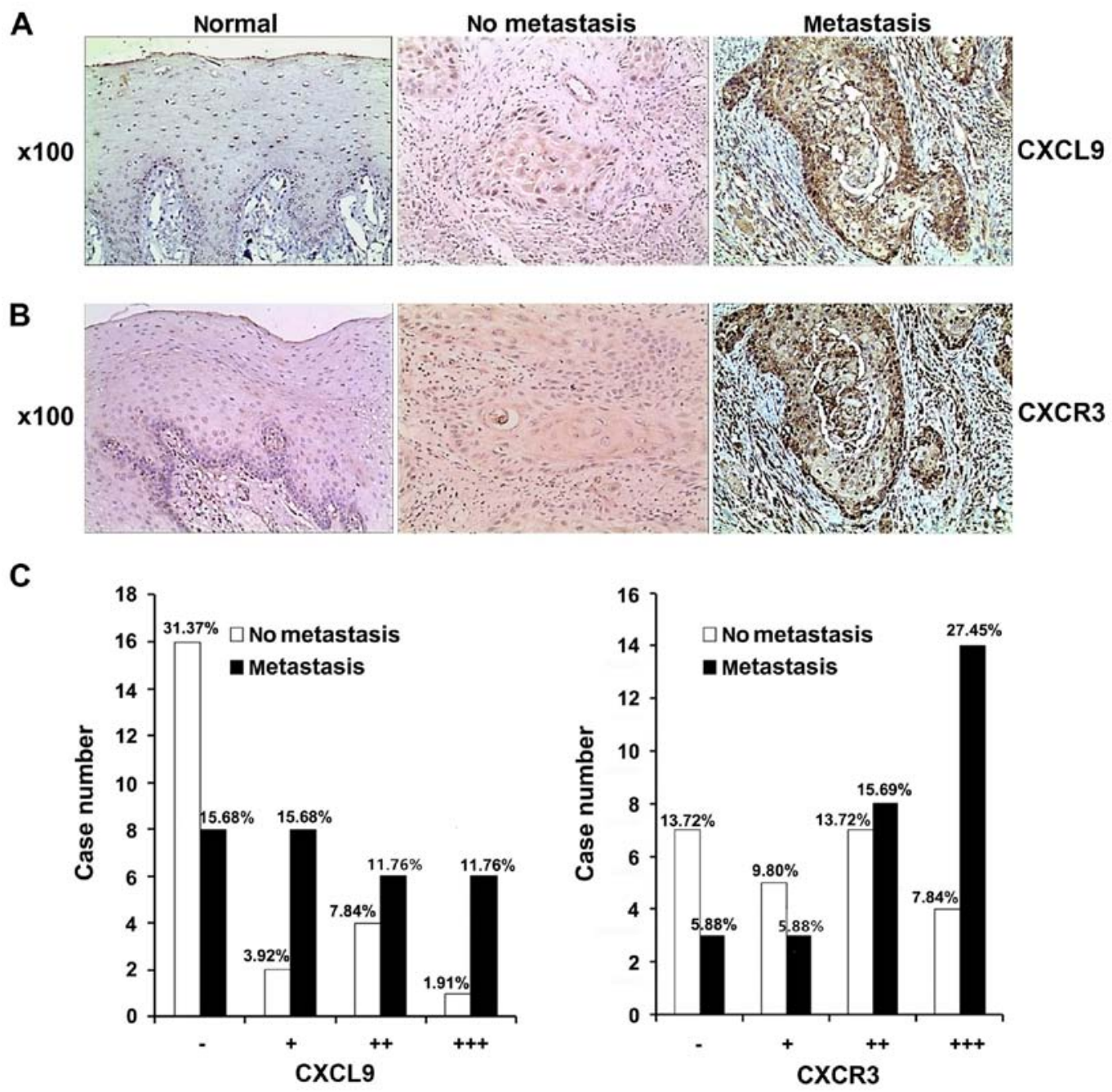

Figure 1. CXCL9 and CXCR3 expression levels in tongue tissue as assessed by immunocytochemical staining (magnification, x100) (normal, and non-lymph node and lymph node metastatic squamous carcinoma tongue tissues). (A) CXCL9 ratings were (-), (+), and (+++), respectively. (B) CXCR3 ratings were (-), (+), and (+++), respectively. (C) Differences in CXCL9/CXCR3 were obtained between non-lymph node and lymph node metastasis tongue squamous carcinoma tissues $(\mathrm{P}<0.05)$.

CXCR3 neutralizing antibodies did not promote Cal-27 cell proliferation. Cells treated for $24 \mathrm{~h}$ with $100 \mathrm{nM} \mathrm{CXCL9}$ and CXCR3 neutralizing antibodies $(2.0 \mu \mathrm{g} / \mathrm{ml})$ or $\operatorname{IgG}(2.0 \mu \mathrm{g} / \mathrm{ml})$ exhibited no statistically significant differences $(\mathrm{P}>0.05)$ in proliferation (Fig. 2C).

CXCR3 overexpression did not affect Cal-27 cell proliferation. Cal-27-GFP and Cal-27-CXCR3 cells were incubated for $24 \mathrm{~h}$ with $100 \mathrm{nM} \mathrm{CXCL9}$, followed by $3 \mathrm{~h}$ of incubation with CCK-8 reagent at room temperature before absorbance assessment at $450 \mathrm{~nm}$. Compared with the control group, cell proliferation exhibited no statistically significant difference (P>0.05) (Fig. 2C).

Low CXCR3 expression was not related to Cal-27 cell proliferation. Cal-27-shRNA-scramble and Cal-27-shRNAhCXCR 3 transfected cells were treated for $24 \mathrm{~h}$ with $100 \mathrm{nM}$ CXCL9, followed by $3 \mathrm{~h}$ of incubation with CCK- 8 reagent at room temperature before absorbance assessment at $450 \mathrm{~nm}$. Compared with the control group, cell proliferation exhibited no statistically significant difference $(\mathrm{P}>0.05)$ (Fig. $2 \mathrm{C})$. The aforementioned data indicated that CXCL9 did not promote Cal-27 cell proliferation.
The CXCL9/CXCR3 axis promotes migration in Cal-27 cells. Cal-27 cells seeded in 6-well plates were cultured for $3 \mathrm{~h}$ with CXCL9 at 0,50,100 and $150 \mathrm{nM}$, respectively; then the cell monolayer was scratched. After $0,12,24$ and $48 \mathrm{~h}$ of incubation, respectively, the samples were imaged, and migration areas were determined. Compared with the control group, a higher migration rate of Cal-27 cells was observed after treatment with various concentrations of CXCL9 (except for 0 and $50 \mathrm{nM}$ after $12 \mathrm{~h}$ ), with statistical significance $(\mathrm{P}<0.05)$ (Fig. 3A). Compared with the CXCL9 group, $100 \mathrm{nM} \mathrm{CXCL9}$ administered in combination with CXCR3 neutralizing antibodies $(2.0 \mu \mathrm{g} / \mathrm{ml})$ and $\operatorname{IgG}(2.0 \mu \mathrm{g} / \mathrm{ml})$ resulted in significantly decreased migration at $48 \mathrm{~h}$, from $93.7 \pm 6.13 \%$ to $83.8 \pm 3.40 \%$ $(\mathrm{P}<0.05)$ (Fig. 3B).

CXCR3 overexpression enhanced CXCL9-stimulating effects on Cal-27 cellmigration.Cal-27-GFPandCal-27-CXCR3 cells were treated for $3 \mathrm{~h}$ with $100 \mathrm{nM}$ CXCL9, followed by scratching. The samples were imaged at $0,12,24$ and $48 \mathrm{~h}$, respectively, to evaluate the migration areas. Compared with the control group, treatment resulted in significantly increased migration areas $(\mathrm{P}<0.05)$ (Fig. 3C). 
A

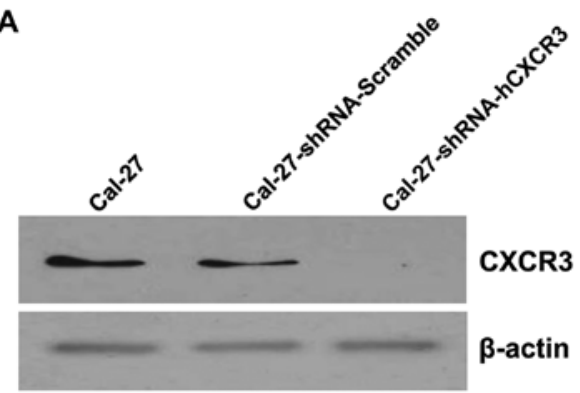

B
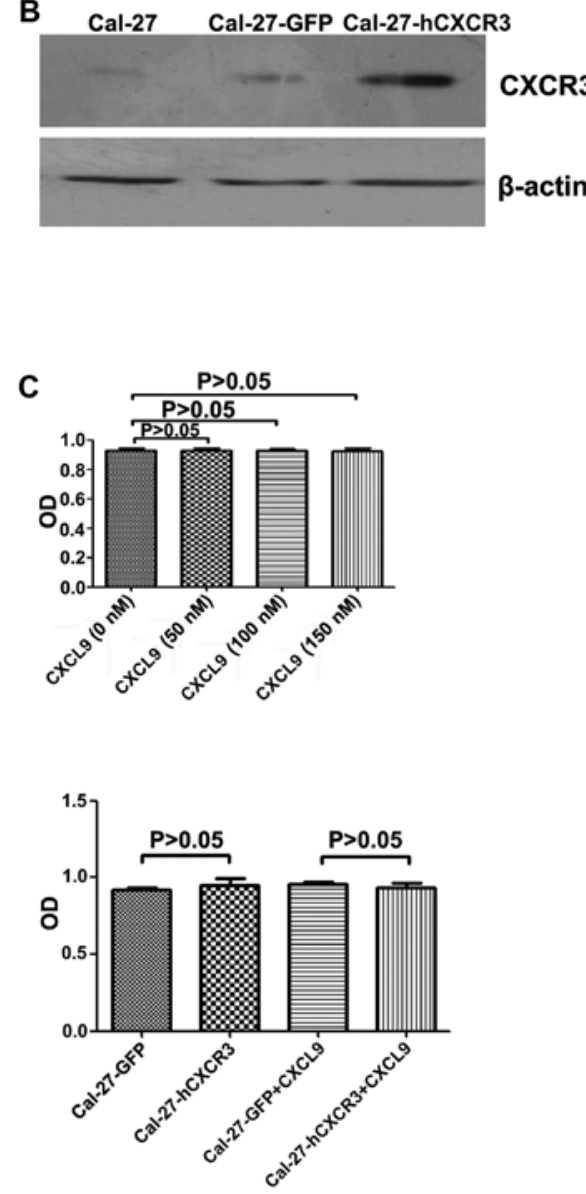
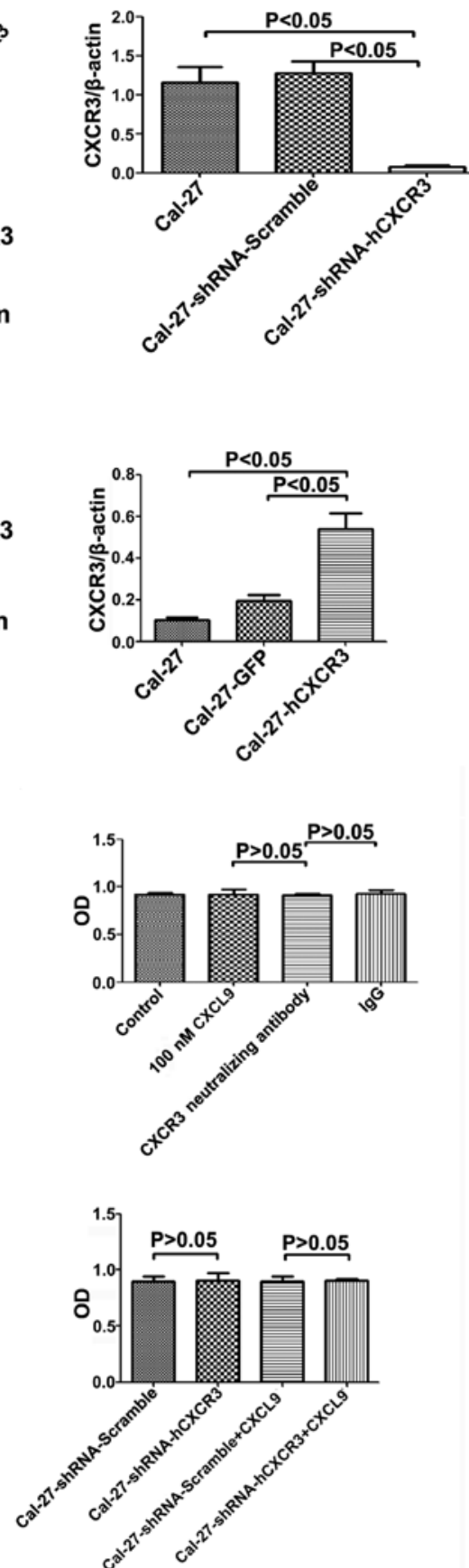

Figure 2. The CXCL9/CXCR3 axis does not promote Cal-27 cell proliferation. (A) Western blotting confirmed that Cal-27 cells were transfected with shRNA$\mathrm{hCXCR} 3$ to inhibit CXCR3 expression $(\mathrm{P}<0.05)$. (B) CXCR3 overexpression in Cal-27 cells transfected with hCXCR3 as assayed by western blotting $(\mathrm{P}<0.05)$. (C) The CCK-8 assay revealed that the CXCL9/CXCR3 axis did not promote Cal-27 cell proliferation with CXCL9 (0, 50, 100 and $150 \mathrm{nM})$, CXCR3 neutralizing antibodies, and after transfection with CXCR3 or shCXCR3.

Low CXCR3 expression attenuated the effect of CXCL9 on Cal-27 cell migration. Cal-27-shRNA-Scramble and Cal-27-shRNA-hCXCR3 cells were scratched and imaged at $0,12,24$, and $48 \mathrm{~h}$ of culture, respectively. Compared with the control group, silencing of CXCR 3 resulted in significantly reduced migration areas $(\mathrm{P}<0.05)($ Fig. 3D). These results revealed that CXCL9 promoted Cal-27 migration, an effect closely related to its receptor CXCR3.

The CXCL9/CXCR3 axis promotes invasion in Cal-27 cells. CXCR3 expressing Cal-27 cells seeded in invasion chambers were cultured for $24 \mathrm{~h}$ with $100 \mathrm{nM}$ CXCL9 in combination with CXCR3 neutralizing antibodies $(2.0 \mu \mathrm{g} / \mathrm{ml})$ or $\operatorname{IgG}(2.0 \mu \mathrm{g} / \mathrm{ml})$. After staining with $0.1 \%$ crystal violet, the specimens were imaged. Compared with the control group, a significant increase in the number of invasive cells was observed $(\mathrm{P}<0.05)$. Compared with the CXCL9 group, treatment with CXCL9 and CXCR3 neutralizing antibodies resulted in significantly reduced cell invasion $(\mathrm{P}<0.05)$ (Fig. 4A).

CXCR3 overexpression positively regulated the inducive effect of CXCL9 on invasion in Cal-27 cells. Cal-27-GFP and 
A

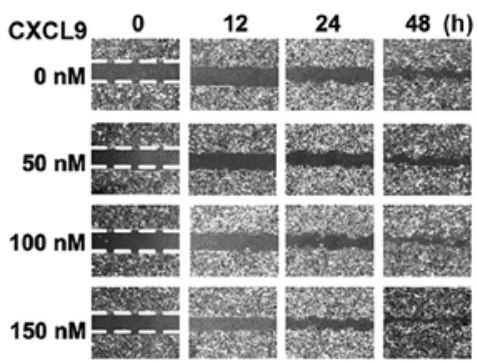

B

C

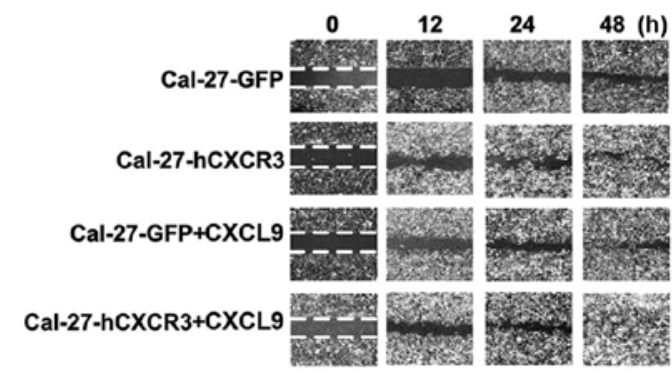

D

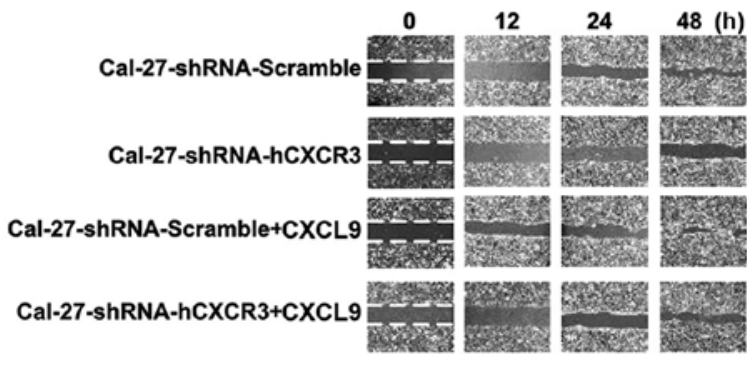

IgG+CXCL9

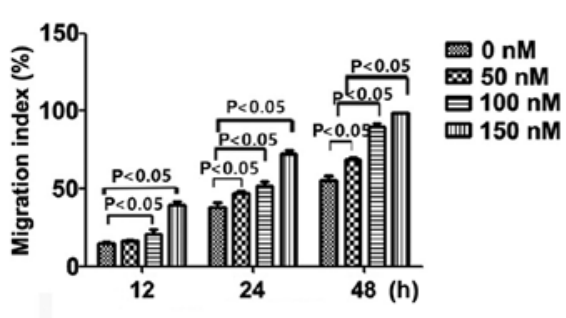

48 (h)
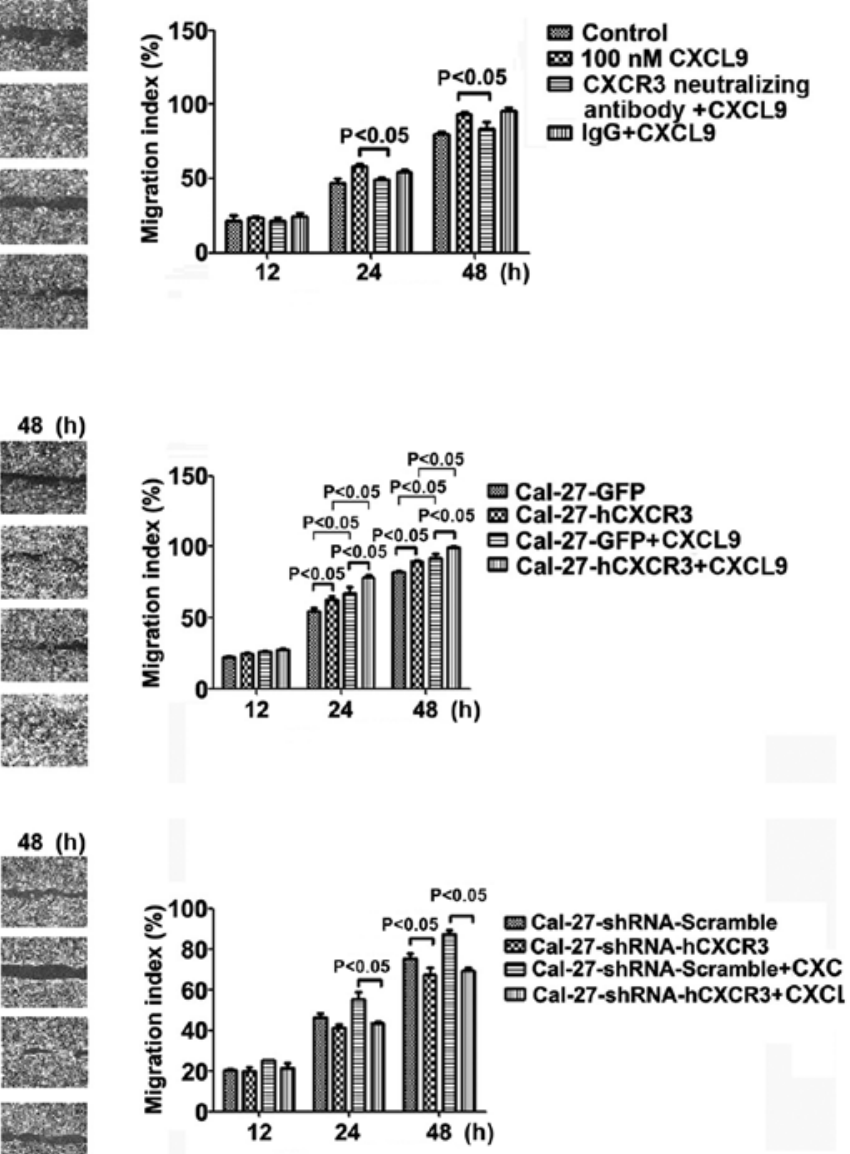

Figure 3. The CXCL9/CXCR3 axis promotes Cal-27 cell migration. (A) Cal-27 cell migration was accelerated by CXCL9 (P<0.05). (B) CXCL9 increased Cal-27 cell migration, which was inhibited by CXCR3 neutralizing antibodies $(\mathrm{P}<0.05)$. (C) Migration of Cal-27-hCXCR3 cells was enhanced compared with that of Cal-27-GFP cells (treated with/without CXCL9) (P<0.05). (D) Mobility of Cal-27-shRNA-hCXCR3 cells was decreased compared with that of Cal-27-shRNA-Scramble cells treated with CXCL9 or without $(\mathrm{P}<0.05)$.

Cal-27-CXCR3 cells were seeded in invasion chambers and treated for $24 \mathrm{~h}$ with $100 \mathrm{nM}$ CXCL9, followed by staining with $0.1 \%$ crystal violet. Compared with the control group, a significant increase in the number of invasive cells was observed $(\mathrm{P}<0.05)$ (Fig. 4B).

Low CXCR3 expression inhibited the inducive effect of CXCL9 on invasion in Cal-27 cells. Cal-27-shRNA-Scramble and Cal-27-shRNA-hCXCR3 cells were seeded in invasion chambers, and treated for $24 \mathrm{~h}$ with $100 \mathrm{nM}$ CXCL9, followed by staining with $0.1 \%$ crystal violet and imaging. Compared with the control group, a significant decrease in the number of invasive cells was observed $(\mathrm{P}<0.05)$ (Fig. 4C). In summary, CXCL9 enhanced the invasive capability of Cal-27 cells, an effect closely related to its receptor CXCR3.
The mix/CXCR3 axis modifies the Cal-27 cytoskeleton. Routinely cultured Cal-27 cells were coated on slides, and incubated for $48 \mathrm{~h}$ with $100 \mathrm{nM}$ CXCL9, CXCR3 neutralizing antibodies $(2.0 \mu \mathrm{g} / \mathrm{ml})$ and $\operatorname{IgG}(2.0 \mu \mathrm{g} / \mathrm{ml})$, and incubated with phalloidin-rhodamine for $60 \mathrm{~min}$ before imaging. Compared with the control group, edge aggregation of F-actin, specifically binding to phalloidin-rhodamine, was evident. Compared with the CXCL9 group, addition of CXCR3 neutralizing antibodies led to significantly decreased edge aggregation of F-actin (Fig. 5A).

CXCR3 overexpression enhanced the inducive effect of CXCL9 on edge aggregation of F-actin. Cal-27-GFP and Cal-27-CXCR3 cells were coated on slides and cultured routinely for $48 \mathrm{~h}$ with CXCL9. After blocking with $1 \%$ 

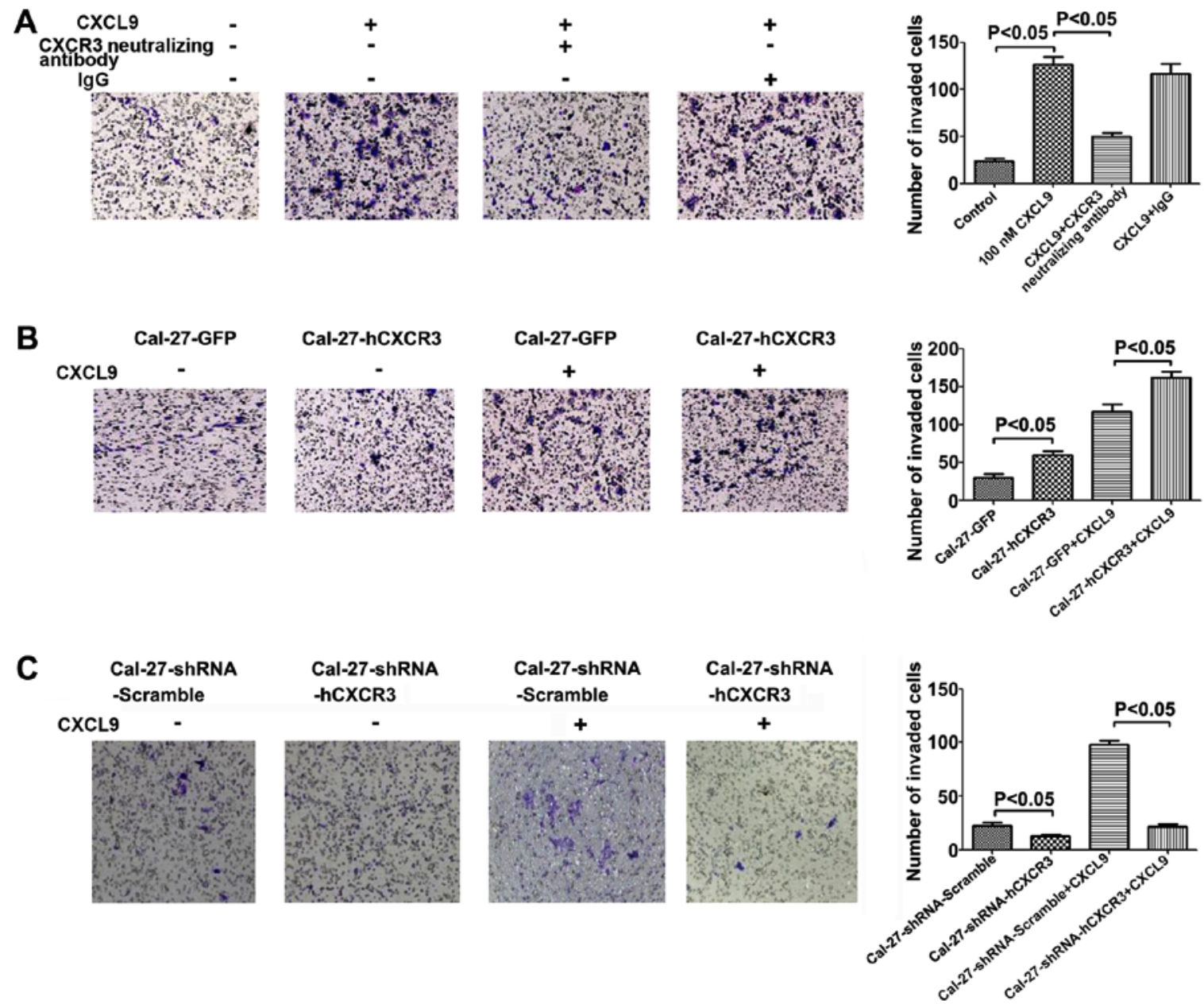

Figure 4. CXCL9/CXCR3 promotes Cal-27 cell invasion. (A) CXCL9 enhanced the infiltrative capability of Cal-27 cells in a cell invasion assay (staining with $0.1 \%$ crystal violet), while CXCR3 neutralizing antibodies attenuated this effect. (B) Infiltration of Cal-27-hCXCR3 cells was enhanced compared with that of Cal-27-GFP cells (both treated with CXCL9). (C) Invasion of Cal-27-shRNA-hCXCR3 cells was decreased compared with that of Cal-27-shRNA-Scramble treated with CXCL9 (both treated with CXCL9). Test results are shown on the right $(\mathrm{P}<0.05)$.

BSA, incubation was performed with rhodamine-labeled phalloidin for $60 \mathrm{~min}$ before imaging. Compared with the Cal-27-GFP group, edge aggregation of F-actin was improved in Cal-27-CXCR3 cells (Fig. 5B).

Reduced CXCR3 expression attenuated the inducive effect of CXCL9 on edge aggregation of F-actin in Cal-27 cells. Cal-27-shRNA-Scramble and Cal-27-shRNA-hCXCR3 cells were seeded on slides and cultured routinely for $48 \mathrm{~h}$ with CXCL9. After blocking with $1 \%$ BSA, incubation was performed with $40 \mu \mathrm{g} / \mathrm{ml}$ rhodamine-labeled phalloidin for $60 \mathrm{~min}$ before imaging. Compared with the shRNAScramble group, edge aggregation of F-actin was reduced in Cal-27-shRNA-hCXCR3 cells (Fig. 5C). Overall, CXCL9 enhanced the edge aggregation of F-actin in Cal-27 cells, an effect closely related to its receptor.

The CXCL9/CXCR3 axis induces EMT in Cal-27 cells. Cal-27 cells from the test and control groups were coated on slides and cultured routinely. Both groups underwent the same process, except for 24-h treatment with $100 \mathrm{nM}$ CXCL9 in the test group. Cells were fixed with paraformaldehyde, permeabilized for 20 min with $0.1 \%$ Triton $\mathrm{X}-100$, blocked for $2 \mathrm{~h}$ in goat serum, and incubated with anti-E-cadherin or anti-vimentin primary antibodies, followed by rhodamine-labeled secondary antibodies and DAPI staining before imaging. Compared with the control group, E-cadherin expression in Cal-27 cells was significantly reduced in the CXCL9 group while vimentin levels were increased. Compared with the CXCL9 group, addition of CXCR3 neutralizing antibodies attenuated the reduction of E-cadherin expression as well as vimentin level increase. For western blotting, Cal-27 cells from the CXCL9 and control groups were lysed, and proteins in the resulting lysates were separated by SDS-PAGE and electrophoretically transferred onto Immobilon-P membranes. The membranes were then incubated with primary antibodies (anti-E-cadherin and anti-vimentin) overnight at $4^{\circ} \mathrm{C}$, followed by $1 \mathrm{~h}$ of incubation with HRP-labeled secondary antibodies at room temperature before chemiluminescent detection. The results were consistent with the aforementioned immunofluorescence findings (Fig. 6A and D).

CXCR3 overexpression enhanced the inducive effect of CXCL9 on expression increase of vimentin and E-cadherin level decrease in Cal-27 cells. Cal-27-GFP and Cal-27-CXCR3 cells were treated for $24 \mathrm{~h}$ with $100 \mathrm{nM}$ CXCL9, fixed with paraformaldehyde and permeabilized for $20 \mathrm{~min}$ with $0.1 \%$ Triton X-100. After blocking for $2 \mathrm{~h}$ in goat serum, the 


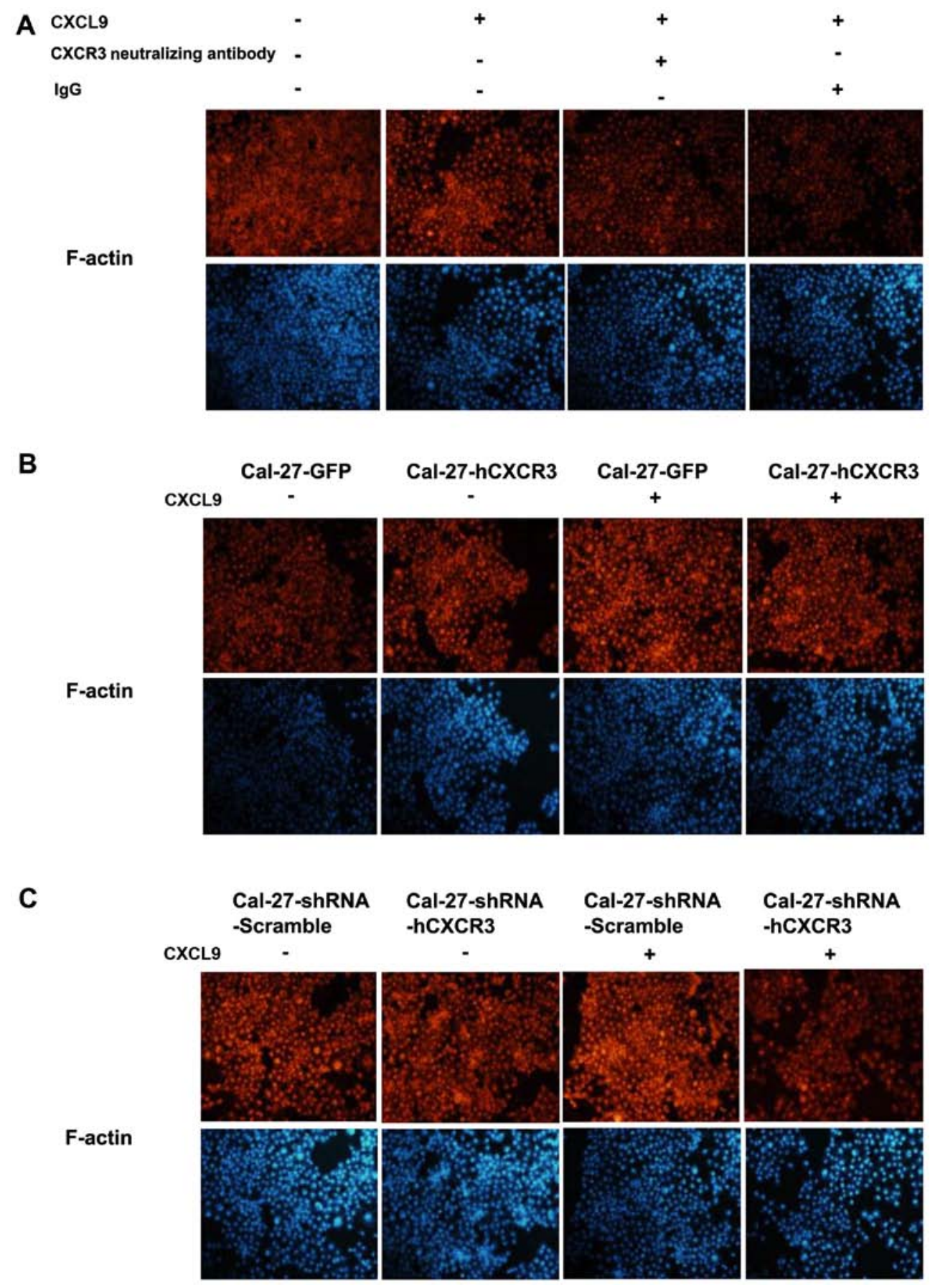

Figure 5. The mix/CXCR3 axis modifies the cytoskeleton in Cal-27 cells. (A) Edge aggregation of F-actin in Cal-27 cells was increased by CXCL9. CXCR3 neutralizing antibodies significantly attenuated such alterations. (B) Aggregation of F-actin in Cal-27-hCXCR3 cells was enhanced (treated with/without CXCL9). (C) Aggregation of F-actin in Cal-27-shRNA-hCXCR3 cells was decreased (treated with/without CXCL9).

samples were sequentially incubated with primary antibodies (anti-E-cadherin or anti-vimentin) and rhodamine-labeled secondary antibodies, followed by DAPI counterstaining before imaging. Compared with the control group, E-cadherin expression in Cal-27 cells was reduced significantly whereas vimentin levels were enhanced. For western blotting, Cal-27-GFP and Cal-27-CXCR3 cells from the CXCL9 and control groups were lysed. Proteins in whole cell lysates were separated by SDS-PAGE, and electrophoretically transferred onto Immobilon-P membranes. The latter were incubated with primary antibodies (anti-E-cadherin and anti-vimentin) overnight at $4^{\circ} \mathrm{C}$, followed by $1 \mathrm{~h}$ of incubation with HRP-labeled secondary antibodies at room temperature before chemiluminescent detection. The results were consistent with the immunofluorescence findings (Fig. 6B and D).

Silencing of CXCR3 attenuated the inducive effect of CXCL9 on E-cadherin level reduction and vimentin expression increase in Cal-27 cells. Cal-27-shRNA-Scramble and Cal-27-shRNA-hCXCR3 cells were treated for $24 \mathrm{~h}$ with $100 \mathrm{nM}$ CXCL9. After sequential incubation with primary antibodies (anti-E-cadherin or anti-vimentin) and rhodaminelabeled secondary antibodies, the samples were treated with DAPI before imaging. Compared with the Scramble group, CXCR3 silencing did not result in E-cadherin reduction in 

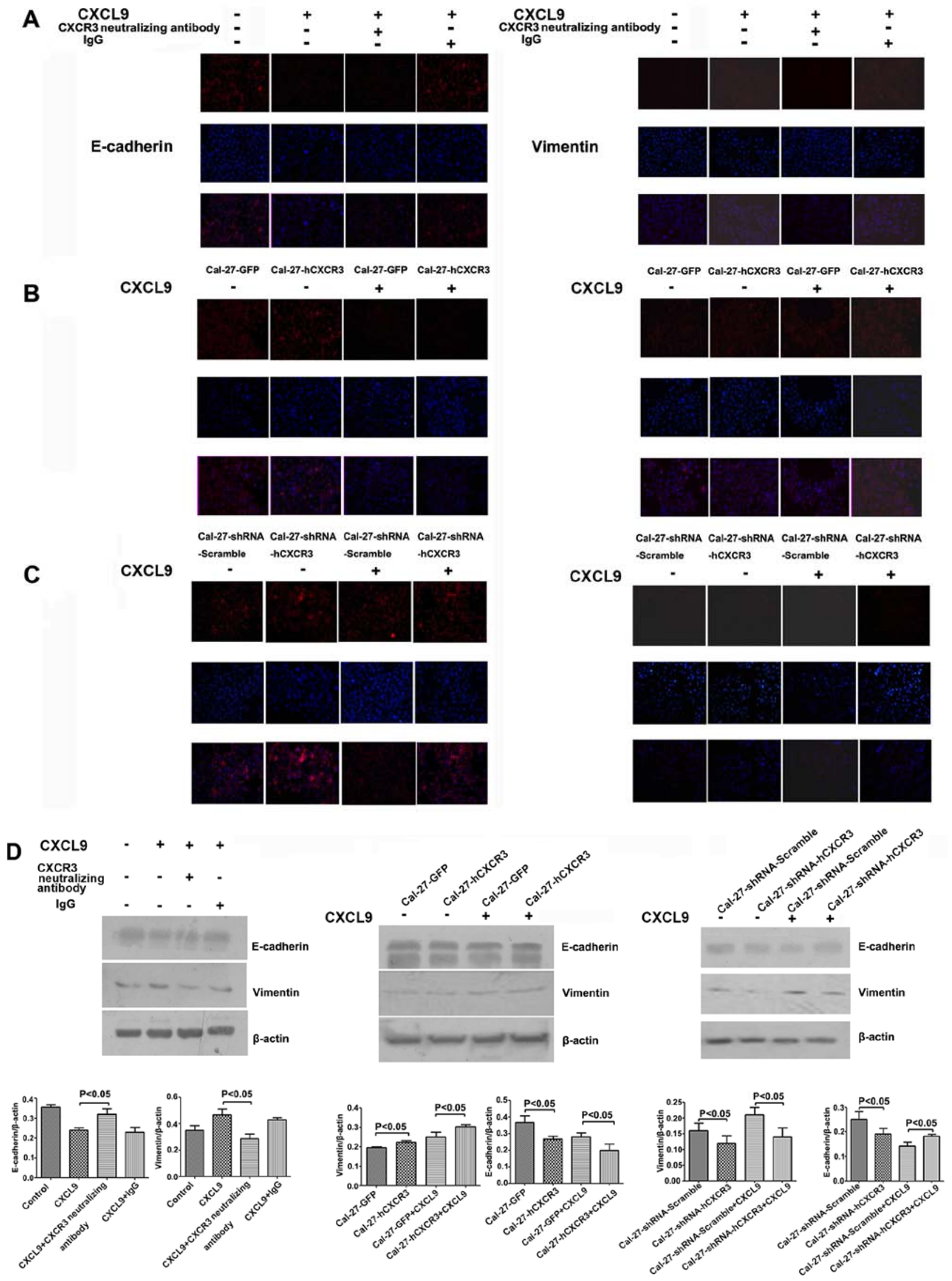

Figure 6. The CXCL9/CXCR3 axis induces EMT in Cal-27 cells. (A) Immunofluorescence of E-cadherin and vimentin in Cal-27 cells with CXCL9 alone or in combination with CXCR3 neutralizing antibodies. E-cadherin expression in Cal-27 cells was significantly reduced in the CXCL9 group while vimentin was increased. CXCR3 neutralizing antibodies attenuated such alterations. (B) Compared with the Cal-27-GFP group, E-cadherin levels were significantly reduced while vimentin was increased in the Cal-27-hCXCR3 group treated with CXCL9. (C) Compared with the Cal-27-shRNA-Scramble group, the expression level of vimentin was lower in the Cal-27-shRNA-hCXCR3 group while the level of E-cadherin exhibited no obvious change. (D) Western blotting of vimentin and E-cadherin protein levels in Cal-27 cells (CXCR3 neutralizing antibodies, CXCR3 overexpression, or CXCR3 knockdown) after treatment with CXCL9. 
A

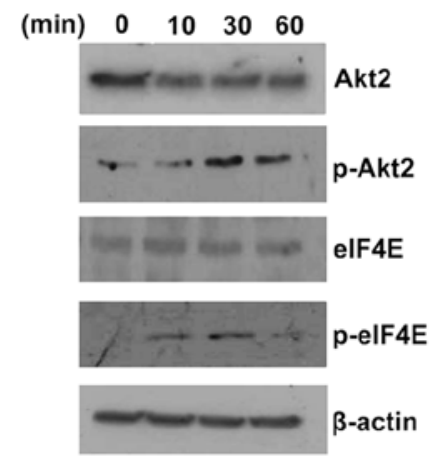

C

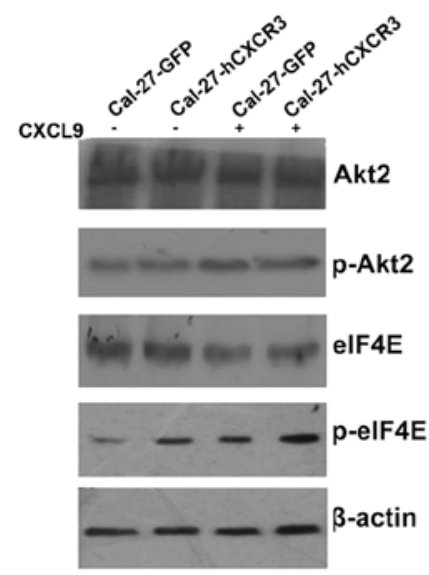

B
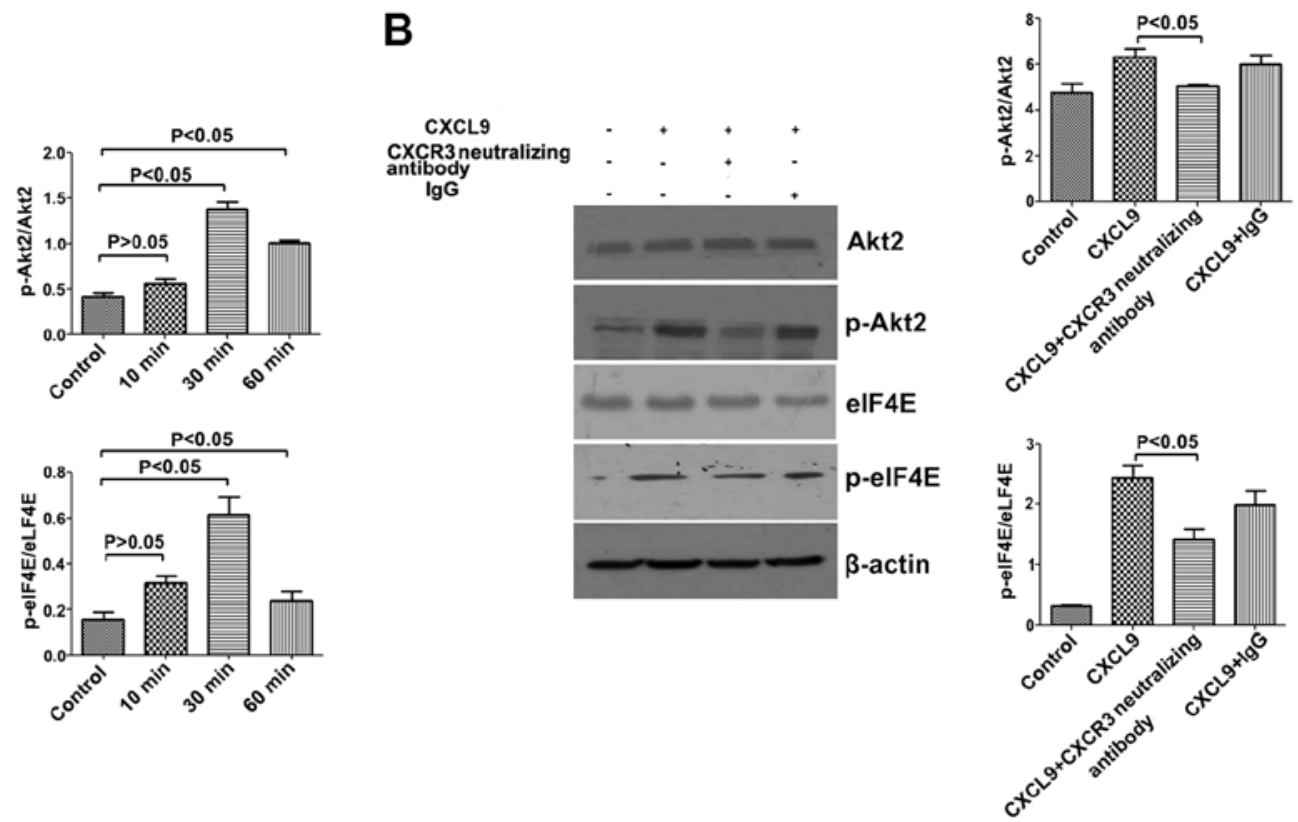

D
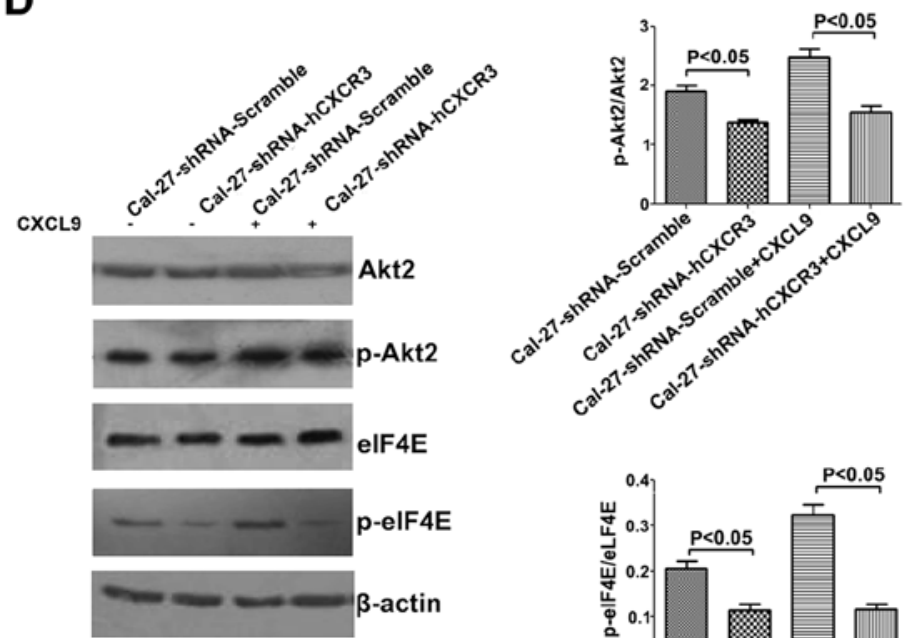
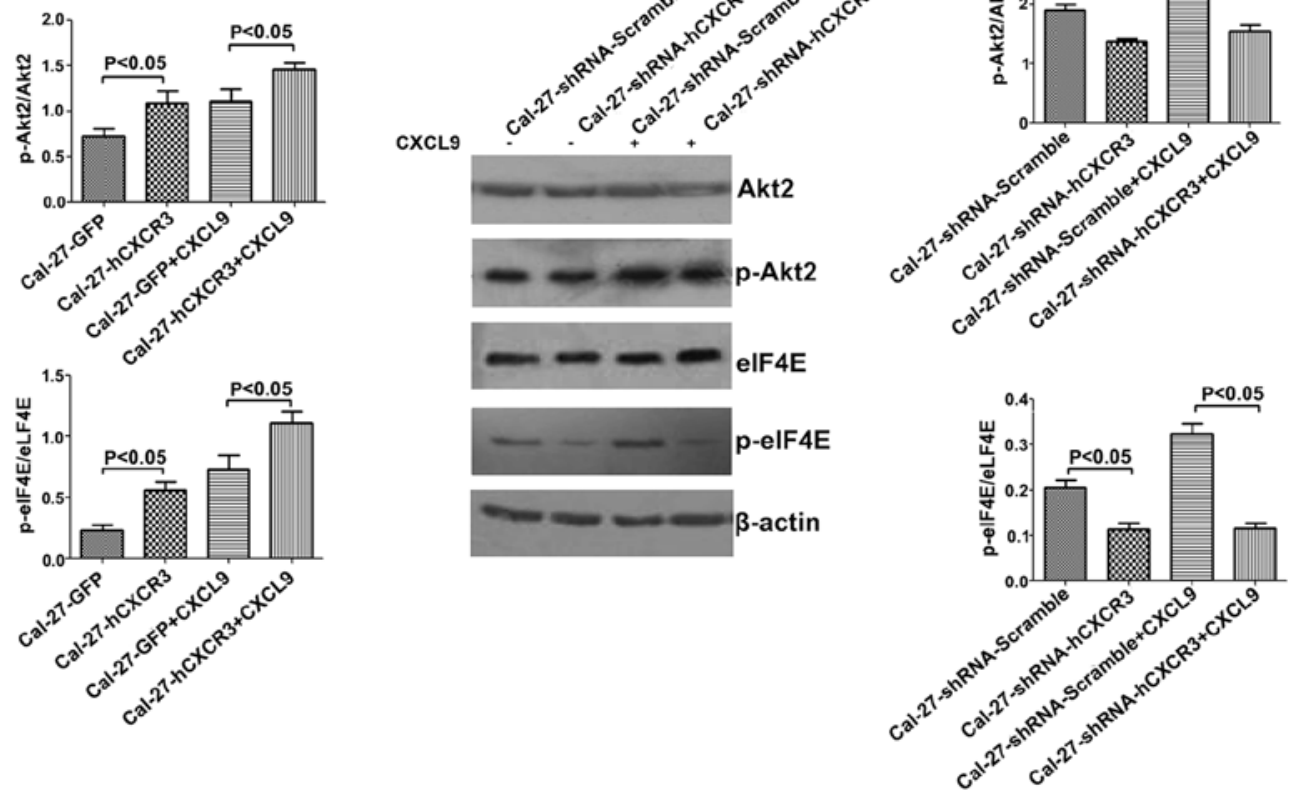

Figure 7. The CXCL9/CXCR3 axis activates Akt signaling in Cal-27 cells. (A) Western blotting of Akt2/p-Akt2 and eIF4E/p-eIF4E protein expression levels in Cal-27 cells treated with $100 \mathrm{nM}$ CXCL9 (10,30 and $60 \mathrm{~min}$ ), with $\beta$-actin used as the loading control. (B) Protein expression levels of Akt2/p-Akt2 and eIF4E/p-eIF4E in Cal-27 cells treated with CXCL9 and CXCR3 neutralizing antibodies $(2.0 \mu \mathrm{g} / \mathrm{ml})$ or without; IgG $(2.0 \mu \mathrm{g} / \mathrm{ml})$ was used as a negative control (C and D) CXCR3 overexpression enhanced the inducive effect of CXCL9 on p-Akt2 and p-eIF4E expression in Cal-27 cells. Conversely, silencing of CXCR3 decreased such effect.

Cal-27 cells but it did decrease the expression level of vimentin. For western blotting, Cal-27-shRNA-Scramble and Cal-27shRNA-hCXCR3 cells of the CXCL9 and control groups were lysed. Proteins in whole cell lysates were then separated by SDS-PAGE and electrophoretically transferred onto Immobilon-P membranes. This was followed by sequential incubation with primary antibodies (anti-E-cadherin or vimentin) overnight at $4^{\circ} \mathrm{C}$, and HRP-labeled secondary antibodies at room temperature $1 \mathrm{~h}$ before chemiluminescent detection. The results were consistent with the immunofluorescence findings (Fig. 6C and D). Hence, in Cal-27 cells, CXCL9 downregulated E-cadherin, an epithelial cell marker, while concomitantly upregulating vimentin, a mesenchymal marker, an activity closely related to its receptor CXCR3.

The CXCL9/CXCR3 axis activates Akt signaling in Cal-27 cells. To further explore how the CXCL9/CXCR3 axis induces EMT in Cal-27, we treated Cal-27 cells with $100 \mathrm{nM}$ CXCL9, and whole cell total protein was extracted at 10, 30, and $60 \mathrm{~min}$, respectively, for western blotting. The results indicated an induction of phosphorylated Akt 2 and eIF4E by CXCL9, which peaked at $30 \mathrm{~min}$, with statistically significant 
differences $(\mathrm{P}<0.05)$ (Fig. 7A). According to the aforementioned findings, the cells were incubated with $100 \mathrm{nM}$ CXCL9, CXCR3 neutralizing antibodies $(2.0 \mu \mathrm{g} / \mathrm{ml})$ and $\mathrm{IgG}$ $(2.0 \mu \mathrm{g} / \mathrm{ml})$. Compared with the CXCL9 group, addition of CXCR3 neutralizing antibodies resulted in decreased relative amounts of p-Akt2 (from $6.28 \pm 0.39$ to $5.01 \pm 0.07$ ) and p-eIF4E (from $2.44 \pm 0.21$ to $1.42 \pm 0.16$ ), with statistically significant differences $(\mathrm{P}<0.05)$ (Fig. 7B).

Moreover, CXCR3 overexpression enhanced the inducive effect of CXCL9 on p-Akt2 and p-eIF4E in Cal-27 cells. Cal-27-GFP and Cal-27-CXCR3 cells were stimulated for 30 min with $100 \mathrm{nM} \mathrm{CXCL9,} \mathrm{and} \mathrm{total} \mathrm{cell} \mathrm{protein} \mathrm{was}$ extracted for western blotting. Compared with the control group, relative amounts of p-Akt 2 in Cal-27-CXCR 3 cells were significantly increased, from $1.11 \pm 0.12$ to $1.46 \pm 0.06$, as well as p-eIF4E, from $0.73 \pm 0.11$ to $1.11 \pm 0.09(\mathrm{P}<0.05)$ (Fig. 7C).

Silencing of CXCR3 attenuated the inducive effect of CXCL9 on p-Akt2 and p-eIF4E in Cal-27 cells. Cal-27shRNA-Scramble and Cal-27-shRNA-hCXCR3 cells were stimulated for $30 \mathrm{~min}$ with $100 \mathrm{nM}$ CXCL9, and total cell protein was extracted for western blotting. Relative amounts of p-Akt 2 were decreased from $2.48 \pm 0.14$ in the control group to $1.55 \pm 0.10$ in the interference group, as well as relative p-eIF4E, from $0.32 \pm 0.03$ to $0.12 \pm 0.02$, with statistically significant intergroup differences $(\mathrm{P}<0.05)$ (Fig. 7D). In summary, the CXCL9/CXCR3 axis upregulated the phosphorylated forms of Akt2 and eIF4E in Cal-27 cells.

\section{Discussion}

TSCC invasion and metastasis is a comprehensive, multifactorial, consecutive, and multistep biological process. The underlying molecular mechanisms remain incompletely understood. However, the critical roles of chemokines in tumor progression are increasingly recognized along with the association of inflammation with tumors. In the tumor microenvironment, paracrine or autocrine chemokines bind to their cognate receptors on the tumor cell surface, induce cytoskeleton rearrangement, and facilitate tight attachment of tumor cells to lymphatic endothelial cells, promoting directional migration (14). The cytoskeleton is highly flexible and restructurable, mainly involving the balance between aggregation and dispersion of cellular F-actin, as the prerequisite for cell movement and migration (15). EMT as cytoskeleton reconstruction, is the primary factor promoting invasion and metastasis in the tumor microenvironment (16). Occurrence of EMT involves alterations of multiple biological behaviors, including the downregulation of the epithelial marker E-cadherin, upregulation of the mesenchymal marker vimentin, and cytoskeleton rearrangement $(17,18)$. Ploenes et al $(19)$ revealed that the chemokine CCL18 promoted invasion and metastasis via EMT in the lung cancer A549 cell line. Another study reported that SDF-1, upon specific binding to its receptor CXCR4, enhanced pancreatic cancer metastasis via EMT (20). Protein levels of the chemokine CXCL9 secreted by carcinoma-associated fibroblasts (CAFs) was significantly higher in TSCC than those of fibroblasts in the normal oral mucosa; meanwhile, the chemokine CXCL9 and its receptor CXCR3 were highly expressed in TSCC tissues, and correlated with cervical LN metastasis (21), suggesting a critical role for CXCL9 and its receptor CXCR3 in tumor invasion and metastasis. The underlying mechanisms remain unknown.

CXCR3 is highly expressed in a wide range of poorly differentiated neoplasms and malignant cells (22-26), including prostate cancer, lung cancer, breast cancer and melanoma, and mediates tumor cell migration and invasion through interaction with its ligand CXCL9 (27). The current study detected high CXCR3 and CXCL9 levels in TSCC tissues, and significant expression differences due to cervical LN metastasis. Moreover, CXCL9 promoted cell migration and invasion in Cal-27 cells. To assess whether the effect of CXCL9 on the tumor Cal-27 cell line was mediated by its receptor CXCR3, CXCR3-overexpressing Cal-27 cells were incubated with CXCL9, and improved migration and invasion was observed. To further confirm the regulatory effect of CXCR3 on Cal-27 cell invasion and metastasis, CXCR3knockdown Cal-27 cells were treated with CXCL9, and a reduction at $48 \mathrm{~h}$ of the migration rate was observed, from $87.2 \pm 5.23 \%$ in controls to $69.4 \pm 7.56 \%$ in the interference group, with a reduction of invasive cells from $98.25 \pm 3.90$ to $21.25 \pm 2.78$, indicating a close correlation between CXCL9 and its receptor CXCR3 in tumor cell migration and invasion, as well as the involvement of the CXCL9/CXCR3 axis in the regulation of tumor cell migration and invasion. Tumor cell migration and invasion is the initial stage of distal metastasis. Ohshima et al (28) demonstrated high CXCR3 expression in gastric cancer with LN metastasis, indicating the possibility that CXCR3 promotes LN metastasis. In a metastatic lymph node melanoma model, Kawada et al (29) detected consecutive expression of CXCR3 in the melanoma B16F10 cell line, and the addition of the ligand CXCL9, CXCL10 or CXCL11 resulted in significantly enhanced cell invasion capability.

In order to explore the specific molecular mechanisms of the CXCL9/CXCR3 axis in inducing TSCC cell migration and invasion, Cal-27 cells were stimulated by CXCL9, followed by staining with rhodamine-labeled phalloidin. As previously revealed, CXCL9 stimulation resulted in edge aggregation of F-actin and cytoskeleton rearrangement. The cytoskeleton is highly flexible and restructurable, mainly involving the balance between the aggregation and dispersion of cellular F-actin, which is the prerequisite for cell movement and migration. Addition of CXCL9 to CXCR3-overexpressing Cal-27 cells led to increased edge aggregation of F-actin and cytoskeleton rearrangement. This effect was not induced by CXCL9 in the CXCR3-knockdown Cal-27 cell line, reflecting the close relationship between CXCL9 and its receptor CXCR3 in tumor cytoskeleton rearrangement, as well as the involvement of CXCL9/CXCR3 in the alteration of the TSCC cytoskeleton. These data corroborated previous findings demonstrating that CXCL12/CXCR4 mediates cytoskeleton rearrangement in intestinal epithelial cells. Cadherin transition is believed to play an important role in tumor cell EMT and cell movement. In malignant tumors, especially those poorly differentiated or metastatic, the epithelial marker E-cadherin is markedly downregulated while the mesenchymal marker vimentin is upregulated. The phenotype transition causes loss of intercellular junction in tumor cells, and hence promotes invasion. In the current study, immunofluorescence and western blotting indicated that CXCL9 promoted E-cadherin downregulation 
in Cal-27 cells as well as vimentin upregulation. Concomitant addition of CXCL9 to CXCR3-overexpressing Cal-27 cells resulted in significantly reduced E-cadherin and increased vimentin, while no obvious expression changes of the two markers were observed in CXCR3-knockdown Cal-27 cells following CXCL9 treatment, suggesting that the regulatory effect of CXCL9 on EMT phenotype is closely related to its receptor CXCR3, with the CXCL9/CXCR3 axis mediating EMT occurrence in TSCC.

In agreement with Li et al (30) who demonstrated that the chemokine CXCL12/CXCR3 axis modulated EMT in liver cancer, the present study revealed the CXCL9/CXCR3 axis mediated EMT in TSCC. Binding of CXCL9 with CXCR3 likely activated the tumor cytosolic Akt signaling pathway, and induced EMT and cytoskeleton rearrangement, hence mediating tumor invasion and metastasis. We stimulated Cal-27 cells with $100 \mathrm{nM}$ CXCL9, and extracted total protein for western blot at 10,30 and $60 \mathrm{~min}$, respectively. As previously revealed, Akt2 and eIF4E phosphorylation was observed, peaking at $30 \mathrm{~min}$ of treatment, corroborating Andersson et al and Zhang et al $(31,32)$. To explore the association of Akt signaling with CXCR function, CXCR3 neutralizing antibodies were applied to block CXCR3 activation. Notably, antibody addition decreased phosphorylated Akt 2 levels $(\mathrm{P}<0.05)$, indicating that Akt2 may be downstream of CXCR3. Moreover, a CXCR3overexpressing cell line was established to explore whether CXCR3 modulated biological behaviors in TSCC cells via the Akt signaling pathway. Addition of CXCL9 to these cells resulted in increased $\mathrm{p}-\mathrm{Akt}-2$, with higher relative amounts from $0.73 \pm 0.11$ to $1.11 \pm 0.09$. To further assess the effect of CXCR3 on the Akt signaling pathway, a CXCR3-knockdown tumor cell line was generated.

As previously revealed, silencing of CXCR3 resulted in reduced phosphorylated levels of Akt2, indicating that CXCL9/CXCR3 likely regulated Cal-27 cell migration, invasion and EMT via Akt 2 signaling. Prior studies revealed close associations of aberrant Akt 2 phosphorylation with tumor proliferation, progression, invasion and metastasis (33), and considered Akt 2 the main connection between CXCR3 and its downstream molecules. Ma et al (26) revealed close associations of CXCR3 with the $\mathrm{G}$ protein subunits Gai and Gaq, with $\mathrm{G}$ protein being the key molecule in Akt activation. In glioma cells, via the Gaq subunit, CXCR3 activated $\mathrm{PI} 3 \mathrm{~K} / \mathrm{Akt}$ to increase intracellular calcium ion levels, thus promoting cell proliferation and chemotaxis (34). In addition, the chemokine receptor CXCR3 modulated cell migration and angiogenesis via Akt (35). A study by Guan et al hinted that CXCR3 regulated osteocarcinoma-related pain via the PI3K/Akt pathway (36).

eIF4E is highly expressed in most human malignancies and positively correlated to tumor invasion and metastasis. Sunavala-Dossabhoy et al (37) reported high expression of eIF4E in head and neck squamous cell carcinoma (HNSCC), with no overexpression in normal tissues and benign neoplasms, suggesting an essential role for eIF4E in the entire process of tumor development. As a downstream effector molecule of Akt signaling, eIF4E phosphorylation is the main initiator of related biological functions. In the current study, stimulation of TSCC cells with CXCL9 induced eIF4E phosphorylation in Cal-27 cells, while addition of CXCR3 neutralizing antibodies resulted in reduced eIF4E levels by blocking CXCR3 activity. Moreover, stimulation of the CXCR3-overexpressing cell line with CXCL9 enhanced eIF4E phosphorylation. To further evaluate the regulatory effect of CXCR3 on eIF4E phosphorylation, a CXCR3knockdown Cal-27 cell line was established. As previously revealed, CXCR3 knockdown attenuated the inducive effect of CXCL9 on eIF4E phosphorylation, indicating a close relationship between CXCL9 and its receptor CXCR3 in the regulation of eIF4E phosphorylation in tumor cells. As a survival promoting signal, eIF4E alters the transcription levels of malignant neoplasm-related mRNAs in various processes, including cell mitosis, oncogene activation, autocrine improvement, cell survival, apoptosis resistance, and communication with the extracellular environment (38).

In summary, the CXCL9/CXCR3 axis is capable of activating Akt signaling in TSCC cells, inducing EMT and cytoskeleton rearrangement, and promoting cancer cell invasion and metastasis.

Three spliced isoforms of CXCR3 receptors has been described, including CXCR3-A (classic CXCR3), CXCR3-B and CXCR3-alt. CXCR3-A is known as a pro-tumor receptor whereas CXCR3-B exhibits antitumor properties (39). Meanwhile, CXCR3 splice variants and its ligands (CXCL9, CXCL10, CXCL11) can activate different signaling pathways in different diseases (40). In the present study, we focused on the mechanisms of the CXCL9/CXCR3 axis promoting invasion and metastasis in tongue squamous cell carcinoma. Notably, the investigation of the functions of other CXCR3 variants and their different ligands in the development and progression of TSCC are warranted in a future study.

The chemokine CXCL9 and its receptor CXCR3 are closely related to migration and invasion in TSCC cells, possibly via ligand binding with its receptor, activating cytosolic Akt signaling, and inducing EMT and skeleton rearrangement, thus mediating tumor cell invasion and metastasis. The present study provided new insights into the role of the CXCL9/CXCR3 axis in inducing cell EMT as well as invasion and metastasis regulation in TSCC. In addition, we elucidated the molecular mechanism of tumor cell invasion and metastasis in relation to chemokines, providing a new basis for clinical prevention and treatment in oral cancer.

\section{Acknowledgements}

We thank Professor Tiejun Li (Peking University Stomatological Hospital) for providing clinical specimens. This study was supported by the National Natural Science Foundation of China (grant nos. 81660448 and 81360401), the Special Health Technical Personnel Training program of Yunnan, China (grant no. L-201612), and the Natural Science Foundation of Yunnan, China (grant no. 2017FE468-006).

\section{References}

1. Kademani D, Bell RB, Schmidt BL, Blanchaert R, Fernandes R, Lambert P and Tucker WM; American Association of Oral and Maxillofacial Surgeons Task Force on Oral Cancer: Oral and maxillofacial surgeons treating oral cancer: A preliminary report from the American Association of Oral and Maxillofacial Surgeons Task Force on Oral Cancer. J Oral Maxillofac Surg 66: 2151-2157, 2008. 
2. Kamangar F, Dores GM and Anderson WF: Patterns of cancer incidence, mortality, and prevalence across five continents: Defining priorities to reduce cancer disparities in different geographic regions of the world. J Clin Oncol 24: 2137-2150, 2006.

3. Yang YF, Guo M and Zhang J: Nursing of tissue defect after radical resection of tongue cancer with forearm flap transplantation. Chin J Aesthetic Med 21: 514-515, 2012 (In Chinese).

4. Landis SH, Murray T, Bolden S and Wingo PA: Cancer statistics, 1999. CA Cancer J Clin 49: 8-31, 1999.

5. Steeg PS: Tumor metastasis: Mechanistic insights and clinical challenges. Nat Med 12: 895-904, 2006.

6. Deryugina EI and Quigley JP: Matrix metalloproteinases and tumor metastasis. Cancer Metastasis Rev 25: 9-34, 2006.

7. Balkwill F: Cancer and the chemokine network. Nat Rev Cancer 4: 540-550, 2004

8. Zlotnik A and Yoshie O: Chemokines: A new classification system and their role in immunity. Immunity 12: 121-127, 2000.

9. Rollins BJ: Chemokines. Blood 90: 909-928, 1997.

10. Strieter RM, Burdick MD, Mestas J, Gomperts B, Keane MP and Belperio JA: Cancer CXC chemokine networks and tumour angiogenesis. Eur J Cancer 42: 768-778, 2006.

11. Vandercappellen J, Van Damme J and Struyf S: The role of CXC chemokines and their receptors in cancer. Cancer Lett 267: 226-244, 2008

12. Liu L, Callahan MK, Huang D and Ransohoff RM: Chemokine receptor CXCR3: An unexpected enigma. Curr Top Dev Biol 68: 149-181, 2005

13. Bodnar RJ, Yates CC and Wells A: IP-10 blocks vascular endothelial growth factor-induced endothelial cell motility and tube formation via inhibition of calpain. Circ Res 98: 617-625, 2006.

14. Friedl $P$ and Alexander S: Cancer invasion and the microenvironment: Plasticity and reciprocity. Cell 147: 992-1009, 2011.

15. Savagner P: Epithelial-mesenchymal transitions: From cell plasticity to concept elasticity. Curr Top Dev Biol 112: 273-300, 2015

16. Jung HY, Fattet L and Yang J: Molecular pathways: Linking tumor microenvironment to epithelial-mesenchymal transition in metastasis. Clin Cancer Res 21: 962-968, 2015.

17. Nakaya Y and Sheng G: EMT in developmental morphogenesis. Cancer Lett 341: 9-15, 2013.

18. Tsai JH and Yang J: Epithelial-mesenchymal plasticity in carcinoma metastasis. Genes Dev 27: 2192-2206, 2013.

19. Ploenes T, Scholtes B, Krohn A, Burger M, Passlick B, MüllerQuernheim J and Zissel G: CC-chemokine ligand 18 induces epithelial to mesenchymal transition in lung cancer A549 cells and elevates the invasive potential. PLoS One 8: e53068, 2013.

20. Li X, Ma Q, Xu Q, Liu H, Lei J, Duan W, Bhat K, Wang F, Wu E and Wang Z: SDF-1/CXCR4 signaling induces pancreatic cancer cell invasion and epithelial-mesenchymal transition in vitro through non-canonical activation of Hedgehog pathway. Cancer Lett 322: 169-176, 2012.

21. Shao S: The role of chemokine Mig and CXCR3 in invasion and metastasis of oral squamous cell carcinoma of tongue. Kunming Medical University, 2013 (In Chinese).

22. Li L, Chen J, Lu ZH, Yu SN, Luo YF, Zhao WG, Ma YH and Jia CW: Significance of chemokine receptor CXCR3 expression in breast cancer. Zhonghua Bing Li Xue Za Zhi 40: 85-88, 2011 (In Chinese).

23. Wu Q, Dhir R and Wells A: Altered CXCR3 isoform expression regulates prostate cancer cell migration and invasion. Mol Cancer 11: 3, 2012.

24. Jenkins MH, Brinckerhoff $\mathrm{CE}$ and Mullins DW: CXCR3 signaling in BRAFWT melanoma increases IL-8 expression and tumorigenicity. PLoS One 10: e0121140, 2015.
25. Li Y, Reader JC, Ma X, Kundu N, Kochel T and Fulton AM: Divergent roles of CXCR3 isoforms in promoting cancer stem-like cell survival and metastasis. Breast Cancer Res Treat 149: 403-415, 2015.

26. Ma B, Khazali A and Wells A: CXCR3 in carcinoma progression. Histol Histopathol 30: 781-792, 2015.

27. Amatschek S, Lucas R, Eger A, Pflueger M, Hundsberger H, Knoll C, Grosse-Kracht S, Schuett W, Koszik F, Maurer D, et al: CXCL9 induces chemotaxis, chemorepulsion and endothelial barrier disruption through CXCR3-mediated activation of melanoma cells. Br J Cancer 104: 469-479, 2011.

28. Ohshima K, Suefuji H, Karube K, Hamasaki M, Hatano B, Tutiya T, Yamaguchi T, Suzuki K, Suzumiya J and Kikuchi M: Expression of chemokine receptor CXCR3 and its ligand, mig, in gastric and thyroid marginal zone lymphomas. Possible migration and autocrine mechanism. Leuk Lymphoma 44: 329-336, 2003.

29. Kawada K, Sonoshita M, Sakashita H, Takabayashi A, Yamaoka Y, Manabe T, Inaba K, Minato N, Oshima M and Taketo MM: Pivotal role of CXCR3 in melanoma cell metastasis to lymph nodes. Cancer Res 64: 4010-4017, 2004.

30. Li X, Li P, Chang Y, Xu Q, Wu Z, Ma Q and Wang Z: The SDF-1/ CXCR4 axis induces epithelial-mesenchymal transition in hepatocellular carcinoma. Mol Cell Biochem 392: 77-84, 2014.

31. Andersson K and Sundler R: Posttranscriptional regulation of TNFalpha expression via eukaryotic initiation factor 4E (eIF4E) phosphorylation in mouse macrophages. Cytokine 33: 52-57, 2006.

32. Zhang B, Ma Y, Guo H, Sun B, Niu R, Ying G and Zhang N: Akt2 is required for macrophage chemotaxis. Eur J Immunol 39 894-901, 2009.

33. Pu R, Li WM, Liu D, Mo XM, Tang Y, Liu LX and Chen BJ: The expression and clinical significance of AKT2, phosphorylated AKT2 in non-small cell lung cancer. Sichuan Da Xue Xue Bao Yi Xue Ban 41: 586-589, 2010 (In Chinese).

34. Maru SV, Holloway KA, Flynn G, Lancashire CL, Loughlin AJ, Male DK and Romero IA: Chemokine production and chemokine receptor expression by human glioma cells: Role of CXCL10 in tumour cell proliferation. J Neuroimmunol 199: 35-45, 2008

35. Willox I, Mirkina I, Westwick J and Ward SG: Evidence for PI3K-dependent CXCR3 agonist-induced degranulation of human cord blood-derived mast cells. Mol Immunol 47: 2367-2377, 2010.

36. Guan XH, Fu QC, Shi D, Bu HL, Song ZP, Xiong BR, Shu B, Xiang HB, Xu B, Manyande A, et al: Activation of spinal chemokine receptor CXCR3 mediates bone cancer pain through an Akt-ERK crosstalk pathway in rats. Exp Neurol 263: 39-49, 2015.

37. Sunavala-Dossabhoy G, Palaniyandi S, Clark C, Nathan CO, Abreo FW and Caldito G: Analysis of eIF4E and 4EBP1 mRNAs in head and neck cancer. Laryngoscope 121: 2136-2141, 2011.

38. Siddiqui N and Sonenberg N: Signalling to eIF4E in cancer. Biochem Soc Trans 43: 763-772, 2015.

39. Boyé K, Billottet C, Pujol N, Alves ID and Bikfalvi A: Ligand activation induces different conformational changes in CXCR3 receptor isoforms as evidenced by plasmon waveguide resonance (PWR). Sci Rep 7: 10703, 2017.

40. Berchiche YA and Sakmar TP: CXC chemokine receptor 3 alternative splice variants selectively activate different signaling pathways. Mol Pharmacol 90: 483-495, 2016. 